\title{
Multistage Decision Making in Bimodal Information Environment
}

\author{
R. A. Aliev ${ }^{1,2,3(\bowtie)}$ \\ 1 Georgia State University, Atlanta, USA \\ raliev@asoa.edu.az \\ 2 Azerbaijan State Oil and Industry University, 20 Azadlig Avenue, AZ1010 Baku, Azerbaijan \\ 3 Department of Information Systems Engineering, Near East University, Lefkosa, North Cyprus
}

\begin{abstract}
Multistage decision making finds its important applications in various fields. A series of works devoted to solving deterministic, stochastic and fuzzy multistage problems by using dynamic programming exist. It is needed to account for the fact that real-world multistage problems are characterized by partially reliable information. Unfortunately, up to day, no works on multistage decision making problems under bimodal information exist. In this work, we consider multistage problem under Z-number-valued information. States, control actions and transition law are described by Z-number-valued restrictions and relations. An optimal solution is found as one which allows to arrive to a predefined Z-number-valued goal. A numerical example is considered for analysis of validity of the proposed approach.
\end{abstract}

